\title{
MODELO DE COMPORTAMIENTO DE MATERIALES ANTE FUERZAS MECANICAS
}

JIMENEZ ALEIXANDRE, $M^{*} P$. Instituto Castelao. Vigo.

\section{SUMMARY}

This experience was carried out with students in Secondary School as a model of behaviour of different materials exposed to mechanical forces, and their variation under heating and water exposure. The aim is not only the acquisition of knowledge, but mainly the way sludents learn.

\section{INTRODUCCION}

Uno de los problemas que marca nuestra práctica docente es si los alumnos aprenden realmente, o, expresado en otros términos, si la adquisición de nueva información va acompañada de la integración de esta información a su correspondiente marco conceptual. Cuando esta incorporación no se produce, el alumno es capaz de repetir una definición, pero no de aplicar el conocimiento adquirido a situaciones nuevas. El aprendizaje no ha sido significativo. (Novak, 1982).

Sin duda existe una estrecha vinculación entre la adquisición de conocimientos y destrezas, y la metodologia empleada en el proceso docente (Gil, 1983). Por nuestra parte intentamos emplear una metodología activa, en la que el alumno se ejercite en algunas habilidades cientificas -observación, comparación, - clasificación... - y en la que la discusión de las experiencias en clase juega un papel fundamental.

La experiencia que se detalla sirve de introducción en ler. curso de B.U.P. a la tectónica. Se llevó a cabo en los cursos 82-83 y 83-84 con todos los grupos de $1^{\circ}$ del Instituto Castelao.

\section{PRE-TEST}

Como introducción al tema se sometió a los alumnos a un pre-test (anónimo) relativo a la unidad de Geodinámica interna (Anexo 1), igual que hacemos al comienzo de cada unidad. Los objetivos de esta prueba son:

- Averiguar el estado de los conocimientos de los alumnos y en particular la persistencia de errores que corresponden a una percepción intuitiva de la realidad. (Valera et al 1983).

- Suscitar una discusión en clase sobre las teorias catastrofistas y actualistas y sobre la tectónica de placas.

- Despertar su interés y provocar el planteamiento de preguntas, ya que (Giordan, 1982) «Sólo la pregunta claramente formulada puede conferir a la solución el estatuto de respuestan; asi como la formulación de hipótesis.

Los resultados estadísticos en el curso 83-84 se exponen en la Tabla 1. Los comentaremos brevemente, no sin señalar que somos conscientes de las limitaciones de esta experiencia y que los resultados presentaban un cierto margen de variación de unos grupos a otros $(210$ alumnos, 6 grupos).

Pregunta 1. El 15\% de los alumnos mantiene opiniones "catastrofistas". Respecto a los "actualistas", la discusión en clase evidencia que admiten la acción, más conspicua, de los agentes externos y la destrucción del relieve, pero no la de los agentes internos, estando generalizada la idea de que el relieve no puede formarse por causas actuales. Esto nos sugiere, para cursos posteriores, sustituir d por una formulación en la que se establezca esta distinción. Refuerza esta hipótesis el alto porcentaje (82) de respuestas correctas a la pregunta 2.

Pregunta 3. Relacionada con la experiencia que nos ocupa. La respuesta correcta es marcada por el $64 \%$. Además de $19^{\prime} 5 \%$ (suma de $a+b$ ) que da respucstas unilaterales, existe un $15 \%$ para los que las rocas «nunca se pliegan".

ENSENANZA DE I.AS CIENCIAS, 1985, pP. 10R-112 


\begin{tabular}{|c|c|c|c|c|c|c|c|}
\hline cuest. & $x$ & cuest. 2 & $x$ & \multicolumn{2}{|c|}{ cuest. $3 \$$} & \multicolumn{2}{|c|}{ cuest. $4 \pi$} \\
\hline$a$ & 15 & a & 2 & a & 9 & $\mathbf{a}$ & 21 \\
\hline$b^{*}$ & 73 & b & 8 & $b$ & 10.5 & $b^{*}$ & 69 \\
\hline c & 1 & $c^{\cdot}$ & 82 & $c^{\circ}$ & 64 & c & 8.5 \\
\hline d & 9 & $d$ & 6 & d & 15 & d & 1.5 \\
\hline$n \cdot \dot{c}$ & 2 & n.c. & 2 & $\mathbf{n} \cdot \mathbf{c}$. & 1.5 & & \\
\hline$\overline{\mathbf{p}}$ & 27 & $P$ & 18 & $P$ & 36 & $\mathbf{P}$ & 31 \\
\hline $\mathbf{s}$ & 3.1 & $s$ & 2.6 & $s$ & $3 \cdot 3$ & s & 3.2 \\
\hline
\end{tabular}

Tabla I.- Porcentajes de respuestas al Pre-test. No 210 . P en la suma de respuestas erróneas y n.c.; y smdesviacton otandard.

Pregunta 4. Senalaremos la existencia de un 22 '5\% de fijistas (suma de a + d).

Asi como posteriormente a la discusión en clase de las preguntas 1 y 2 -en prácticamente sin intervención del profesor los alumnos "actualistas» tienen suficientes argumentos para convencer a los "catastrofistas" proponemos a los alumnos que enuncien con sus propias palabras el principio del Actualismo; en la discusión de la pregunta 3, el profesor no interviene para apuntar la solución, sino que propone una experiencia sobre el comportamiento de distintos materiales. Queda claramente explícito que no es posible réalizarla con rocas y que será un modelo de comportamiento.

\section{DESARROLLO DE LA EXPERIENCIA}

Previamente el profesor proporciona a jos alumnos las uefiniciones, ya estudiadas en cursos anteriores de com: portamiento elástico, plástico y rigido.

Cada alumno tiene un guión de trabajo (Tabla II) y el cuaderno de notas, y cada equipo de 4 alumnos una serie de los 10 objetos que van a utilizar y un mechero de alcohol.

Los alumnos deben ir realizando las pruebas descritas en el guión y anotando el resultado en la casilla correspondiente. Finalmente, como conclusiones de cada equipo, responden a las preguntas del guión. (Es fact tible la realización de todo este proceso en una horal

Es parte indispensable de la experiencia la discusión en clase de los resultados. La razón es, aparte de la importancia didáctica del proceso de discusión, que algunos de los objetivos que pretendemos sólo se alcan. zarán si los alumnos comprueban (o, mejor, descubren) que unos equipos obtienen resultados diferentes de otros.

Veamos por ejemplo la discusión en $1^{\circ} \mathrm{D}$ :

$\rightarrow$ La cinta del caucho $\left(n^{\circ} 1\right)$ es elástica para todos, pero la plastilina ( $n^{\circ} 2$ ) es calificada por 6 equipos como plástica en todas las pruebas, y por 3 equjpos como rigida y frágil en la prueba $A$ (antes de calentarla con las manos). Uno de estos 3 equipos anotó en el cuaderno

«Al principio es plástica, pero al llegar a cierto li. mite se rompió. Depende de la fuerza que se haga." Por supuesto el profesor evita pronunciarse sobre el resultado «correcton.

- El portaobjetos ( $n^{\circ} 3$ ) es rigido para 8 equipos y uno señala que además es frágil: lo rompieron. Una vez más algunos señalan en la discusión que el resultado depende de la fuerza. El cubreobjetos fue roto por 3 equipos en la prueba $A\left(n^{\circ} 4\right)$.

- La varilla hueca de vidrio ( $n^{\circ} 5$ ) y el clavo obtienen resultados uniformes - la varilla rígida en frio y plástica en caliente y el clavo siempre rígido-; en la cinta de $\operatorname{zinc}\left(n^{\circ} 7\right)$ vuelven a estar divididos: para $S$ equipos es elástica en la prueba $B$ (compresion) y para los otros 4 plástica.

Durante la discusión sobre estos resultados súbitamente se hace la luz para una alumna que casi grita

“;Pero entonces cualquier material se puede comportar como elástico, plástico o rigido según las condiciones y según la fuerza que se haga!".

Subrayemos el paralelismo de esta formulación con la de un especialista en tectónica: Mattauer (1976).

"Los cuerpos reales, y en particular las rocas, no son nunca perfectamente elásticos, plásticos o viscosos (...) los cuerpos se comportan sucesivamente en el curso de la deformación como elásticos, plásticos y viscosos."

- También hay acuerdo entre todos los grupos acer. ca de la rigidez de la escayola seca y la plasticidad de la húmeda.

Por último se discuten en clase las conclusiones, las contestaciones a las preguntas del guión y sus posibles correcciones, a la luz de lo debatido anteriormente. 
TABLA II

Experiencia de Geologla

COMPOFTAMIENTO DE LOS MATERIALES

Indituto

FRENTE A LAS FUERZAS MECANYCAS

Castelso

INFORMACION:tr material ante un esfuerzo decimos que se comporta a) ELASTICAMENTE o que es ELASTICO at al actuar ol esfuerzo sobre bl se deforma y al dejar de actuar el esflerzo recupera su forma. b) PLASTICAMENTE o que es PLASTICO a al actuar el esfuerzo sobre 61 se defora $y$ al dejar de actuar no recupera su forma primitiva. c) RIGIDAMENTE o que es RIGIDO si no se deforma.Si el espuerzo es muy grande se puede llegar a romper (Decimos que es fRuGIL). Los materiales geológicos plísticos originan al deformarse estructuras llamada PLIEGUES.Los matertales geologlcos rigtdos *1 romperse orfiginan estructuras llamadas fructuRAs.

PRocedTMENTO: somete a los 10 objetos a las pruebas que enunciamos - continuación y hax un cuadro con los resultados, marcando E los de comportamiento ELASTICO, $P$ a los de comportamiento PLASTICO, R a LOS RIGIDOS Y $F$ a los FRAGILES.

PRUEBAS: A) Estira el objeto, intentando aumentar su longftud

(Fuerza de tracción

B) Sujeta el objeto por un extremo y empuja por el otro,tratando de acortarlo. (Fuerza de compresión $\rightarrow-\rightarrow+\cdots$ )

C) Calienta el objeto con las manos y prueba a estirarlo como en $A$.

D) $" n$ " acortarlo como en $B$.

E) Calienta los objetos 5 y 6 a la llama del mechero y prueba como $A$.

F) $" n n " n$ " $n$ como $B$.

G) Con la parte postcrior del lápiz presiona el objeto fuertemente.

\begin{tabular}{|c|c|c|c|c|c|c|c|c|}
\hline PBJETOS $\quad$ PRUEBAS $\rightarrow$ & $\bar{A}$ & B & C & $\mathrm{D}$ & $\vec{E}$ & $F$ & G & otra \\
\hline 1. Cinta de gons (caucho) & & & & & & & & \\
\hline L. Plastilina & & & & & & & & \\
\hline 3. Portaobjetos de vidrio & & & & & & & & \\
\hline 4. Cubreobjetos de vidrío & & & & & & & & \\
\hline S.Varilla hueca de vidrio & & & & & & & & \\
\hline 6. Clavo de hierro & & & & & & & & \\
\hline 7. Cinta de zinc & & & & & & & & \\
\hline 8. Cinta de hojalata & & & & & & & & \\
\hline 9. Escayo1a seca & & & & & & & & \\
\hline Ho. Escayola recien hume & & & & & & & & \\
\hline
\end{tabular}

PREGUNTAS: Según estos resultados clasifics los objetos y los materiales utilizados en Elásticos, Plásticos, Rfgidos y fráglies.

2. Un mismo material ise comporta siempre igual? ique condiciones intrinsecas (longftud, grosor...) pueden alterar su coaportamiento?

3. ¿Qut condiciones externas (temperatura, agua, tienpo de actuación...) pueden alterar ese comportamiento? Pon ejemplos.

4. ¿Como podrfas alterar el comportamiento de 6 y de 7 ?

5. iA que tipo de materiales, por el comportaniento, crees que pertenecen la mayorfa de las rocas? ¿Piensas que hay etertas condiciones en que el comportameinto de las rocas se puede alterar.? Especiffea las condtciones. 


\section{EVALUACION}

Al plantear la elaboración de un modelo que sustituyera a los tradicionalmente empleados para ilustrar la tectónica nos proponiamos:

- Que los alumnos descubrieran que un mismo material no siempre se comporta del mismo modo, sino que la intensidad de la fuerza, o el tipo (tracción o compresión) hace variar su comportamiento.

- Que comprobaran que determinadas condiciones externas alteran el comportamiento de los materiates. Por ejemplo la elevación de temperatura y el agua facilitan la deformación.

- Que manipulasen los materiales y llegasen a conclusiones por si mismos, y tras el debate en el equi. po $y$ en clase.

\subsection{Evaluación de los resultados académicos}

Para la evaluación de esta unidad, igual que para otras, utilizamos: el análisis de los cuadernos de clase, pruebas prácticas, de interpretación de diapositivas, etc. y pruebas escritas. Un comentario detallado de los cuadernos y las pruebas prácticas excede el marco de este articulo. Detallaremos, en cuanio a los resultados de las pruebas escritas, las respuestas a las preguntas relacionadas con el Pre-test y la experiencia aqui reseñada. (Ver Anexo $n^{\circ} 2$ ).

Las respuestas correctas a las preguntas 1, 2 o 3 sobre las causas actuales totalizan un $\mathbf{8 5 \%}$.

Las respuestas a la pregunta 4 sobre estructuras orogenicas son:

$\begin{array}{lr}\text { Correctas } & 78 \% \\ \text { unilaterales } & 10 ' 5 \% \\ \text { erróneas } & 45 \% \\ \text { no contestan } & 7.2 \%\end{array}$

Señalaremos que uno de los motivos que nos llevó a diseñar esta experiencia fue, precisamente, el gran nu. mero de respuestas erróneas (entre el 40 y el $50 \%$ ) a esta pregunta registrada en cursos anteriores.

La desviacion entre el $50-60 \%$ que la respondían correctamente durante los cursos $79-80$ y $80-81$ y el $64 \%$ que la responde correctamente en el Pre-test suponemos que se explica a) por tratarse de grupos diferentes en anos distintos; b) porque el Pre-test es una prueba de elección múltiple en la que no tienen que redactar ellos y c) por la situación sicológica del alumno, distinta en una prueba anónima y en un ejercicio que conlleva calificación.

Las respuestas correctas a la pregunta 5 eran el $70 \%$ y a las preguntas 607 el $81 \%$.

El sistema de recuperación inciuye la realización (utilizando material de consulta) de ejercicios como los del Anexo $n^{\circ} 5$. Para los textos ver Holmes (1962) y Dercourt et al (1978).

\subsection{Deficiencias observadas y aspectos a mejorar}

En cursos próximos nos proponemos mejorar este diseño, corrigiendo las deficiencias que nos han sido set̃aladas por algunos compañeros, principalmente:

- Señalar el tiempo durante el que se debe aplicar la fuerza (Creemos que $10 \mathrm{seg}$. es adecuado).

- Medir, utilizando dinamómetros, la intensidad de la fuerza de tracción aplicada (que explica, en muchos casos tas diferencias entre los equipos). Con el material de que disponemos no nos resulta posible medir la de compresión, aunque hemos solicjtado la ayuda de los físicos del Centro.

- Sustituir la escayola por arcilla, ya que la primera, al fraguar en breves momentos, distorsiona los resultados.

Como aspecto positivo del diseño valoramos el bajo costo del material, parte de él de desecho (caucho de una cámara de neumático, tira de hojalata de embalajes, etc.) lo que facilita su montaje en cualquier centro.

\section{REFERENCIAS BIBLIOGRAFICAS}

ANGUITA, F., MORENO, F., 1977, Geologia, procesos internos (Zaragoza, Edelvives).

DERCOURT, J., PAQUET, J., 1978, Geologia (Barcelona, Reverté).

GIL PÉRE2, D., 1983, Tres paradigmas básicos en la Ensehanza de las Ciencias. Enserianza de las Ciencias, Vol. I. n' 1, pp. 26-33.

GIORDAN, A., 1982, La Enseñanzo de las Ciencias (Ma. drid, Pablo del Río-Siglo XXI).
HOLMES, A., 1962, Geologio física (Barcelona, Omega). MATTAUER, M., 1976, Las deformaciones de los materiales de la corteza terrestre (Barcelona, Omega).

NOVAK, J., 1982, Teoria y práctica de la Educoción (Ma* drid, Alianza).

VALERA et al, 1983, Intuición e historia de las ciencias en la ensentanza. Enseñanza de las Ciencias, Vol. $1 n^{\circ} 3$, pp. 205-215. 


\section{Anexo 1}

\section{PRE TEST GEODINAMICA}

1. ¿A qué se debe la existencia de montañas en unos sitios y valles en otros?

a. Se formaron en la antigüedad debido a explosiones y cataclismos que tenian lugar en aquellos tiempos y que hoy dia ya no ocurren.

b. Se formaron hace miles de años por causas geológicas parecidas a las que actúan hoy dia (y siguen formándose en la actualidad).

c. Algunos se formaron hace miles de años y otros hace poco tiempo, por causas que los geólogos desconocen.

d. Otra (dila).

2. Las rocas sedimentarias, que contienen materiales procedentes de otras rocas

a. Se estan formando en la actualidad en algunos lugares de la Tierra.

b. Se formaron, junto con otros tipos de rocas en los primeros tiempos de existencia de la Tierra.

c. Algunas empezaron a formarse hace miles de anos y otras se siguen formando en la actualidad.

d. Orra (dila).

3. ¿A qué se debe que unas veces las rocas aparezcan deformadas (plegadas) y otras rotas (falladas o fracturadas).

a. Depende de la intensidad de ia fuerza: si es pequeña los pliega y si es grande los rompe.

b. Depende del tipo de material rocoso: unos se pliegan (son más elásticos o plásticos) y otros se rompen (son más rígidos o frágiles).

c. Depende del tipo de material y de la intensidad de la fuerza.

d. Las rocas nunca se pliegan: sólo se rompen.

4. ¿Cómo ha sido la posición de los continentes, a lo largo de la historia de la Tierra?

a. Los continentes se separaron hace tiempo y ahora tienen una posición fija.

b. Han variado de posición y tamaño contienentes y océanos y siguen variando aún. c. Los continentes se desplazan sobre los octanos y éstos permanecen iguales.

d. La posición ha sido siempre la misma, la que hay hoy día.

Anexo $n^{\circ} 2$

\section{PREGUNTAS DE PRUEBAS ESCRITAS}

(Distintas preguntas sobre un mismo tema corresponden a pruebas de diferentes grupos.)

1. Completa esta frase: "Las transformaciones a que está sometida la superficie de la Tierra se deben a la acción de

que son hoy iguales

Este principio, establecido por Charles Lyell recibe el nombre de

2. El geólogo del siglo XVIIl Hutton es autor de la frase «El presente es la clave del pasado». Explícala en tèrminos geológicos.

3. Completa esta frase: "Las transformaciones que tienen lugar en la Tierra reciben el nombre de procesos ............................. que, si se producen en la superficie se llaman .................... y si se producen en el interior se llaman

Estos procesos se deben a causas

y los que tuvieron lugar en épocas pasadas son ..................................... a los que tienen lugar ahora. Este principio se conoce como Ley

4. Explica por qué razones las rocas, sometidas a una fuerza, unas veces originan pliegues y otras fracturas. ¿Qué nombre comparten?

5. En algunas montañas del interior de España, por ejemplo, Teruel, se encuentran fósiles de conchas marinas. Explica, cronologicamente qué procesos debieron tener lugar para que esto suceda.

6. Haz un resumen de la teoria de la Deriva Continental de Wegener y menciona algunas pruebas que ia apoyan.

7. Explica cómo se formaron los Andes, según la teoria de la Tectónica de Placas. 